\title{
Reconstruction of Superior Vena Cava and Brachiocephalic Vein Invasion After Thymothymectomy: A Report of a Case and Surgical Procedure
}

\section{(1) Ertan Demirdaş1, (1) Hüseyin Sicim1, (1) Hakan Kartal1, (1) Gökhan Erol1, (1) Hakan Işık², (1) Gökhan Arslan1}

${ }^{1}$ University of Health Sciences, Gülhane Training and Research Hospital, Clinic of Cardiovascular Surgery, Ankara, Turkey

2University of Health Sciences, Gülhane Training and Research Hospital, Clinic of Thoracic Surgery, Ankara, Turkey

\begin{abstract}
Thymoma is one of the most common tumor types in anterior mediastinum, and its surgical treatment according to the type and degree of invasion can be very difficult. A 45-year-old woman with myasthenia gravis underwent a thorax tomography, a finding compatible with thymoma was detected and robotic thymectomy was planned. It was returned to sternotomy because of the presence of severe adhesions. After resection, pericardial patchplasty was
\end{abstract}

\section{Introduction}

Thymomas are the most common primary mediastinal neoplasms, but account for about $50 \%$ of all anterior mediastinal tumors ${ }^{(1)}$. Approximately $15 \%$ of myasthenia performed in the superior vena cava and a graft interposition was made between the left brachiocephalic vein and the right atrium auricular. In this case report, we present a vascular reconstruction alternative in tumor surgery with severe environment and vascular tissue invasion.

Keywords: Thymoma, superior vena cava invasion, reconstruction

gravis patients are diagnosed with thymoma ${ }^{(2)}$. Rarely, thymic tumors may cause thrombus, which may cause obstruction by vena cava superior invasion. Determining the appropriate surgical treatment for anterior mediastinal malignancies, especially invasive cases of superior vena

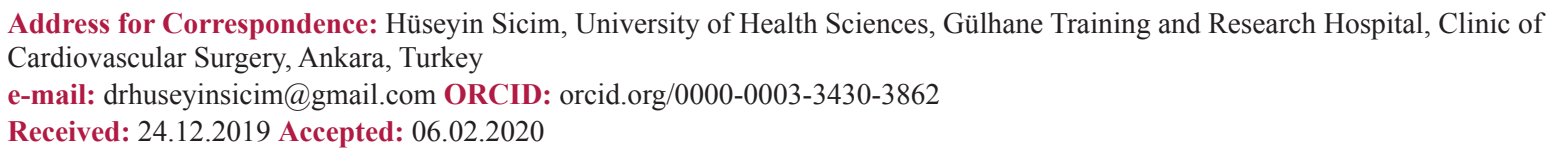

Cite this article as: Demirdaş E, Sicim H, Kartal H, Erol G, Işı1k H, Arslan G. Reconstruction of Superior Vena Cava and Brachiocephalic Vein Invasion After Thymothymectomy: A Report of a Case and Surgical Procedure. EJCM 2020;8(1):59-62.

DOI: 10.32596/ejcm.galenos.2020.12.066 
cava, is a serious problem for surgeons. Various surgical methods can be used to resect the superior vena cava invasive tumors without using cardiopulmonary bypass ${ }^{(3)}$. The type of procedure used depends on the size of the tumor and the extent of invasion to surrounding tissues. Even the most invasive mediastinal tumors can be resected with appropriate surgical techniques without requiring cardiopulmonary bypass. In this case report, we aimed to present a reconstruction of large vascular structures after the resection of the tumor with pericardial, superior vena cava and left brachiocephalic vein invasion.

\section{Case Report}

A 45-year-old female patient was admitted to the hospital with the complaints of double eyelid drop and double vision and she was diagnosed as myasthenia graves 6 months ago. The patient's physical examination was normal and there were no findings of myasthenia graves but acetylcholine receptor antibody was positive $(6.25 \mathrm{mmol} / \mathrm{L})$. Chest X-ray demonstrated an enlarged mediastinum. At control tomography, thymoma-compatible image was detected and the operation decision was given. Computed tomography scan revealed a 4-cm mass at the anterior mediastinum (Figure 1). Robotic thymectomy operation was planned. A $3 \mathrm{~cm}$ mini-thoracotomy was performed at the right anterior axillary line. However, the patient had sternotomy because of the serious invasion of the tumor mass in the pericardium and surrounding tissues. Poststernotomy mass was observed to be highly invasive in the pericardium, superior vena cava and left innominate vein.

The pericardiectomy was performed and the pericardium was opened. The proximal and distal end of the superior vena cava and the left brachiocephalic vein were clamped. The tumor mass was completely resected. The involved parts of the SVC were removed and vascular wall defect was reconstructed with autologous pericardial patch using 5-0 polypropylene (Ethicon Inc., NJ) continuous sutures. The left brachiocephalic vein was transected and total resection was performed. Interposition was performed with a $10 \mathrm{~mm}$ Dacron graft between the left brachiocephalic vein and the right auricle (Figure 2). According to the pathology report, morphological and immunohistochemical findings were consistent with "Type B2 thymoma". The report showed a full-thickness infiltration of the tumor brachiocephalic vein, with focal necrosis $(10 \%)$, and the surgical margins were normal. The patient was discharged on the $8^{\text {th }}$ postoperative day.

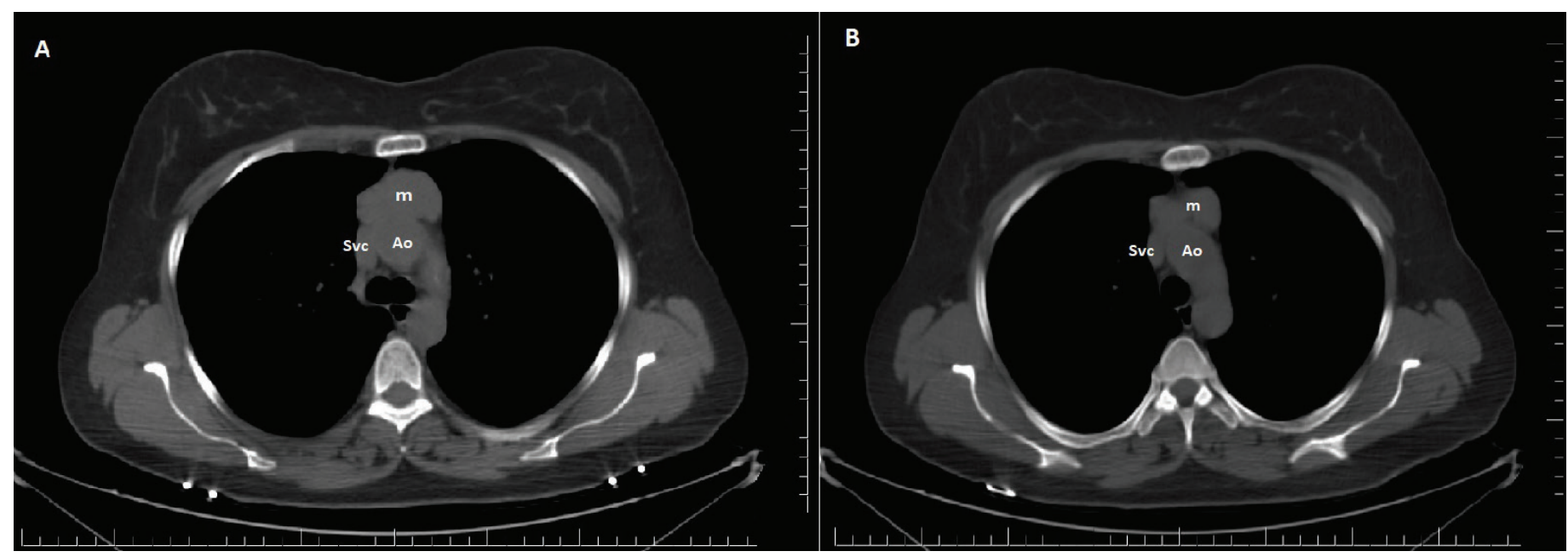

Figure 1. Axial CT image showing anterior mediastinal mass

Ao: Ascending aorta, m: Mediastinal mass, SVC: Superior vena cava, CT: Computed tomography 


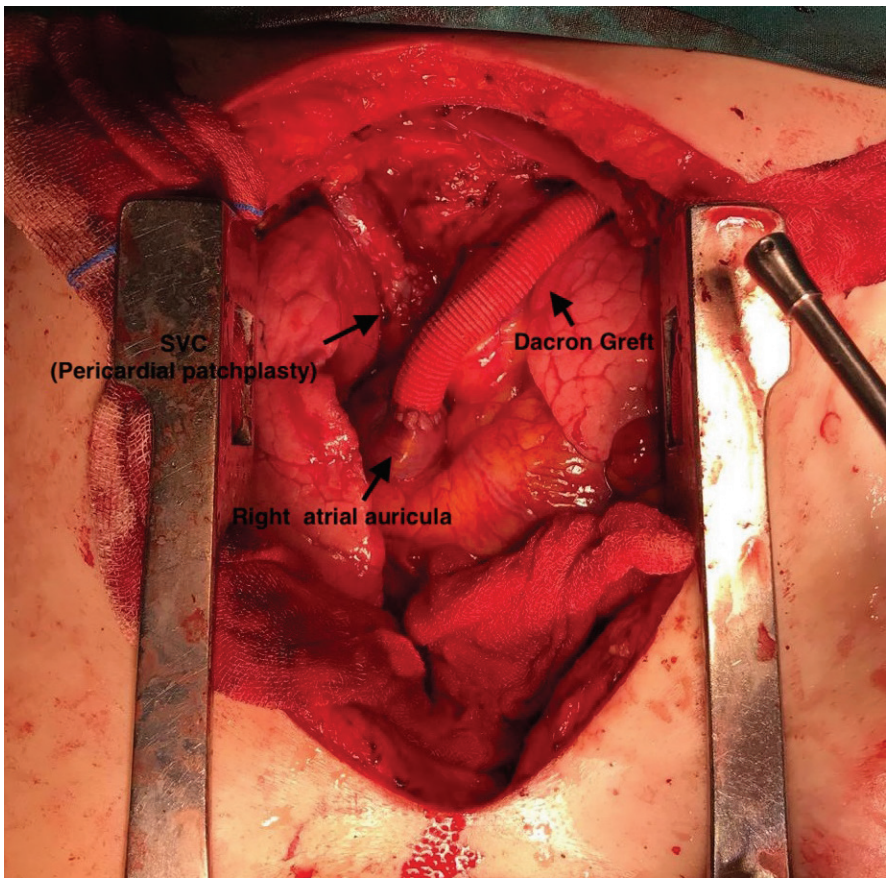

Figure 2. Dacron graft was placed between left subclavian vein and right auricle after resection of the left brachiocephalic vein, pericardial patch was used to reconstruct anterior wall of SVC

SVC: Superior vena cava

\section{Discussion}

Thymoma is a rare neoplasm primarily arising within the anterior mediastinum. Invasive thymomas rarely invade adjacent organs in the mediastinal pleura, usually in the lungs, pericardium, large vessels, and heart ${ }^{(4)}$. Complications of malignant thymomas generally occur as local invasion of adjacent organs. And complications due to myasthenia graves occur secondary, and heart tamponade and SVC syndrome are very rare.

The most appropriate treatment for invasive thymomas is complete resection and therefore, invasive cases require vascular wall reconstruction. It has been reported that SVC is reconstructed by graft replacement of pericardium, saphenous vein, spiral vein graft and polytetrafluoroethylene. The use of spiral ePTFE grafts has been proposed to prevent longer graft patency and compression. Invasive thymoma sometimes progresses quickly and the tumor can spread to the SVC wall through thymic ${ }^{(5)}$. Invasive thymoma usually does not progress like this. Thus, when a tumor in the SVC is associated with a mediastinal tumor, the possibility of an invasive thymoma penetrating thymic vessels should be considered. Thymic branches should be examined in detail to ensure the clearance of tumor mass.

Various surgical approaches can be used depending on the size and extent of the resection of mediastinal tumors. If a total SVC clamp is needed during the operation, the SVC should be clamped at the level above the azygos vein to preserve some side branch circulation to minimize cerebral anoxia. If the clamping is at this level, SVC bypass is not usually required, but if the clamping is below the azygos vein, a procedure that will last longer than 60 minutes, SVC shunt or bypass should be planned $^{(6)}$. In mediastinal tumors, only the need for SVC resection should not be considered as a contraindication for operation. When the SVC is completely resected, the right phrenic nerve usually requires resection; therefore, preoperative pulmonary function testing is essential ${ }^{(7)}$. Where appropriate, complete surgical resection can be performed considering the various surgical techniques described. Different surgical methods can be used to achieve vascular reconstruction. The location, structure, size and degree of invasion of the tumor mass shape the reconstruction. Anastomosis of the left brachiocephalic vein graft interposition on SVC or direct right auricle is controversial.

The use of homograft in surgery is not very common. The graft is not preferred because it is expensive and difficult to obtain. In some publications, there was no significant difference between the patency rates of synthetic grafts and homografts ${ }^{(8)}$. And among the synthetic grafts, PTFE and dacron grafts were the most commonly used grafts, and there was no significant difference between the patency rates of these $\operatorname{grafts}^{(9)}$.

As a result; as seen in this case report, maintaining vascular integrity in superior vena cava and brachiocephalic vein invasion has priority. Techniques such as patchplasty and graft interposition may be used. We would like to 
emphasize that if interposition is performed in large vessel reconstructions such as SVC and brachiocephalic vein, it will contribute to the graft patency rates of the brachiocephalic vein graft interposition anastomosis to the right atrium auricle instead of SVC.

\section{Ethics}

Informed Consent: It was obtained from the patients.

Peer-review: Externally and internally peer-reviewed.

\section{Authorship Contributions}

Surgical and Medical Practices: E.D., Concept: H.K., Design: H.S., Data Collection or Processing: G.E., Analysis or Interpretation: H.I., Literature Search: G.E., Writing: G.A.

Conflict of Interest: No conflict of interest was declared by the authors.

Financial Disclosure: The authors declared that this study received no financial support.

\section{References}

1. Lee HI, Jang IS, Jeon KN, Ko GH, Lee JS, et al. Thymoma and Synchronous Primary Mediastinal Seminomas with Florid Follicular Lymphoid
Hyperplasia in the Anterior Mediastinum: A Case Report and Review of the Literature. J Pathol Transl Med. 2017;51:165-70.

2. Romi F. Thymoma in myasthenia gravis: from diagnosis to treatment. Autoimmune Dis. 2011;2011:474-512.

3. Garcia A, Flores RM. Surgical management of tumors invading the superior vena cava. Ann Thorac Surg. 2008;85:2144-6.

4. Alur I, Taştan H, Tanrısever GY, Emrecan B. A thymoma invading the right atrium. Turk Gogus Kalp Dama 2015;23:371-3.

5. Terada Y, Ono N, Noguchi T, Kamakari K, Kitano M. A case of thymoma protruding into the superior vena cava through the thymic vein. Ann of Thorac Surg. 2004;77:1088-90.

6. Singh S, Sherif H, Reul GJ. Reconstruction of the superior vena cava with the aid of an extraluminal venovenous jugulo-atrial shunt. Tex Heart Inst J. 2000;27:38-42.

7. Yu G, Chen G, Wu S. A large malignant thymomas of the anterior mediastinum. J Thorac Dis. 2015;7:E88-E91.

8. Dortland R.W.H, Leeuwen M.S, Steijling J.J.F, Theodorides Th, van Vroonhoven Th. J.M.V. Long-term results with vein homograft in femorodistal arterial reconstructions. Eur J Vasc Surg 1991;5:557-64.

9. Roll S, Müller-Nordhorn J, Keil T, Scholz H, Eidt D, Greiner W, Willich SN.Dacron vs. PTFE as bypass materials in peripheral vascular surgery-systematic review and meta-analysis. BMC Surg. 2008 Dec 19;8:22. 